Case Study

\title{
Effects of the dual chest banding using elastic bands on the shoulder pain of scapular dyskinesis patient with winging and elevated scapular
}

\author{
WON-Gyu Yoo ${ }^{1)}$ \\ 1) Department of Physical Therapy, College of Biomedical Science and Engineering, Inje University: \\ 607 Obangdong, Gimhae, Gyeongsangnam-do 621-749, Republic of Korea
}

\begin{abstract}
Purpose] The purpose of this study was to investigate the effect of dual chest banding using elastic bands on the shoulder pain of a scapular dyskinesis patient with winging and an elevated scapula. [Subject] The subject was a 40-year-old male scapular dyskinesis patient with winging, an elevated scapula, and shoulder pain. [Methods] This study developed a method of dual chest banding using elastic bands, and the subject applied this method for 1 month [Results] After applying dual chest banding for one month, the scapular position and scapular winging were improved when compared to the initial conditions. The upper trapezius pressure pain threshold was increased. [Conclusion] The present study suggests that dual chest banding provides a mechanical effect and increases proprioception and therefore reduces abnormal scapular mobility. The present study suggests that dual chest banding using elastic bands could be applied to patients with scapular dyskinesis in the clinic.

Key words: Elastic band, Scapular dyskinesis, Upper trapezius pressure pain
\end{abstract}

(This article was submitted Sep. 24, 2015, and was accepted Oct. 31, 2015)

\section{INTRODUCTION}

Recent findings have suggested that postural correction focusing on scapular alignment is an effective way to reduce neck and shoulder pain ${ }^{1,2)}$. Wegner et al. suggested that a postural correction strategy for scapular position was helpful in patients with pain for restoring muscular activation during work at a computer ${ }^{1)}$. Assessment of postural alignment is an important part of orthopedics because impaired postural alignment may cause excessive compression and stress and, consequently, pain involving muscles, joints, and ligaments ${ }^{3}$. When evaluating shoulder problems, it is important to measure the static position of the scapula ${ }^{3,4)}$. Patil and RAO insisted that gait training using a wrapped elastic band could assist dorsiflexion and eversion in the ankle during the swing phase, thus helping to facilitate normalization of the gait pattern ${ }^{5)}$. The elastic band, which provides varied resistance through the range of movement, has been used for rehabilitation in combination with therapeutic exercise ${ }^{6}$. It is light and portable, has low resistance, and can be adjusted to accommodate various situations ${ }^{6}$. The purpose of this study was to investigate the effect of dual chest banding using elastic bands on the shoulder pain of a scapular dyskinesis patient with winging and an elevated scapula.

\section{SUBJECT AND METHODS}

The subject was a 40-year-old male scapular dyskinesis patient with winging, an elevated scapula, shoulder pain, and no specific surgical or medical history of the shoulder. The subject received an explanation about the purpose and methods of the study prior to participation and provided informed consent according to the ethical principles of the Declaration of Helsinki. He had been experiencing severe pain in the right upper trapezius for 6 months when performing detailed assembly work

Corresponding author. Won-gyu Yoo (E-mail: won7y@inje.ac.kr)

(C2016 The Society of Physical Therapy Science. Published by IPEC Inc.

This is an open-access article distributed under the terms of the Creative Commons Attribution Non-Commercial No Derivatives (by-nc-nd) License $<$ http://creativecommons.org/licenses/by-nc-nd/4.0/>. 
and during long periods of computer work. However, he did not undergo any particular treatment. Two physical therapists evaluated the participants for the presence or absence of scapular dyskinesis. The participant was asked to elevate his arms overhead within 3 seconds in a thumbs-up position and to lower them within 3 seconds. A load was attached to the subject according to the subject's weight: $3 \mathrm{lb}$. The subjects performed four repetitions (two for flexion and two for abduction), which were recorded by a video camera. Two physical therapists determined the presence of scapula dyskinesis by consensus. The result of the initial scapular dyskinesis test showed that the right scapular exhibited winging and elevation. This study compared the scapular position and upper trapezius pressure pain between before and after applying dual chest banding using elastic bands. For the first chest band, one end of the elastic band was tied behind the left shoulder and around the chest, and it passed under the right axillae, crossed behind the back and was then tied behind the right shoulder. The first chest band restricted the winging of the scapula by increasing the serratus anterior muscle tension and by the mechanical effect of the elastic band, which was expected to reduce scapular winging. For the second chest band, the elastic band was placed around the chest at the T8-T10 level. The second chest banding restricted the elevation of the scapula by increasing the muscle tension of the latissimus dorsi and lower trapezius. Dual chest banding also provided an additional mechanical effect and increased proprioception for limitation of scapular hypermobility. The subject applied the bands for 1 month when working with his right hand and during long periods of computer work. This study compared the scapular position and upper trapezius pressure pain between before and after applying the bands. An algometer used to determine the amount of pressure applied to the participant by the therapist. A dolorimeter (Fabrication Enterprises, White Plains, NY, USA) pressure algometer was used to measure pressure pain. The dolorimeter consisted of a metal probe that can measure pressures of up to $20 \mathrm{lb}$ in 0.25 $\mathrm{lb}$ increments, respectively. A palpation meter (PALM, Performance Attainment Associates, St. Paul, MN, USA) was used to measure the distance and inclination between two bony landmarks of the body. The PALM consisted of an inclinometer and two caliper arms, and the inclinometer had a semicircular arc that moved within the range $0-30^{\circ}$ in either direction from the midline at $1^{\circ}$ intervals. The PALM was used as a special body-tool interface to combine the advantages of palpation with the objectivity and reliability of caliper and inclinometer measurements. The scapular depression distance was determined using the PALM calculator, which combined the values for distance and inclination between the C7 spinous process and the superior angle of scapula. The horizontal distance from the superior angle to the spinal process was defined as follows: one arm of the caliper was fixed at the superior angle of the scapula, and the other arm was positioned at the spinal process such that the inclinometer indicated $0^{\circ}$. The scapular winging angle was determined as the vertical angle between the spinal process and medial border of the scapula.

\section{RESULTS}

After one month of applying dual chest banding, the scapular depression distance $(3.2 \mathrm{~cm})$ was increased when compared with the initial value $(1.5 \mathrm{~cm})$. Furthermore, the scapular winging angle (22 degrees) was decreased when compared with the initial value (26 degrees), and the upper trapezius pressure pain threshold (4.2 lb) was increased when compared with initial value $(2.3 \mathrm{lb})$.

\section{DISCUSSION}

Although the subject had scapular dyskinesis with scapular winging or tipping while elevating or lowering the arm, he did not have severe pain or functional limitation in daily life ${ }^{7)}$. Madeson et al. reported that the prevalence of scapular dyskinesis increased with cumulative swimming training in a pain-free population, which indicates that scapular dyskinesis can appear in a pain-free population and may be caused by low muscular endurance ${ }^{8)}$. Although scapular dyskinesis can occur in a pain-free population, the scapular kinematics of the dyskinesis are reported to be similar to those of subjects with impingement syndrome ${ }^{9)}$. Therefore, scapular dyskinesis might be a risk factor for shoulder disorders ${ }^{7)}$. The purpose of this study was to investigate the effect of dual chest banding using elastic band on the shoulder pain of a scapular dyskinesis patient with winging and an elevated scapula. The results showed that after dual chest banding, the scapular position and scapular winging were improved when compared with the initial conditions. The upper trapezius pressure pain threshold was also increased. The serratus anterior originates from the lateral aspects of the upper eight ribs and inserts on the anteromedial border and inferior angle of the scapula ${ }^{4}$. Thus, it positions the scapula closely against the thorax and provides stability to scapular kinematics for prevention of scapular winging 4 ). Weakness of the serratus anterior and altered activation of the upper trapezius can result in abnormal scapular winging and elevation and lead to shoulder impingement syndrome due to an inappropriate position of the glenoid fossa during arm elevation ${ }^{7)}$. Both the latissimus dorsi and lower trapezius contribute to depression of the shoulder girdle, but the insertion was different that the latissimus dorsi for the medical border of scapulae, and latissimus dorsi for the lesser tubercle of humerus ${ }^{4}$. I thought that the first chest band would restrict the winging of the scapula by increasing the serratus anterior muscle tension and by the mechanical effect of the elastic band, which was expected to reduce scapular winging and that the second chest band would restrict the elevation of scapula by increasing the muscle tension of the latissimus dorsi and lower trapezius. Furthermore, I thought that dual chest banding would also provide a mechanical effect and increase proprioception and therefore reduce abnormal scapular mobility. So, this study suggests that dual chest banding using elastic bands could be applied to patient with scapular dyskinesis in the clinic. 


\section{REFERENCES}

1) Wegner S, Jull G, O'Leary S, et al.: The effect of a scapular postural correction strategy on trapezius activity in patients with neck pain. Man Ther, 2010, 15: 562-566. [Medline] [CrossRef]

2) Ha SM, Kwon OY, Yi CH, et al.: Effects of passive correction of scapular position on pain, proprioception, and range of motion in neck-pain patients with bilateral scapular downward-rotation syndrome. Man Ther, 2011, 16: 585-589. [Medline] [CrossRef]

3) Page P, Frank CC, Lardner R: Assessment and treatment of muscle imbalance: The Janda approach. Champaign: Human Kinetics, 2010.

4) Kendall FP, McCreary EK, Provance PG, et al.: Muscles: Testing and Function, with Posture and Pain, 5th ed. Baltimore: Lippincott Williams \& Wilkins, 2005.

5) Patil P, Rao S: Effects of Thera-Band ${ }^{\circledR}$ elastic resistance-assisted gait training in stroke patients: a pilot study. Eur $\mathbf{J}$ Phys Rehabil Med, 2011, 47: 427-433. [Medline]

6) Yoo IG, Yoo WG: The effect of a new neck support tying method using Thera-band on cervical ROM and shoulder muscle pain after overhead work. J Phys Ther Sci, 2013, 25: 843-844. [Medline] [CrossRef]

7) Neumann DA: Kinesiology of the Musculoskeletal System. New York: Mosby, 2002.

8) Madsen PH, Bak K, Jensen S, et al.: Training induces scapular dyskinesis in pain-free competitive swimmers: a reliability and observational study. Clin J Sport Med, 2011, 21: 109-113. [Medline] [CrossRef]

9) McClure P, Tate AR, Kareha S, et al.: A clinical method for identifying scapular dyskinesis, part 1: reliability. J Athl Train, 2009, 44: 160-164. [Medline] [CrossRef] 